\title{
A INFLUÊNCIA DA GOVERNANÇA CORPORATIVA E DO AUDITOR NA DIVULGAÇÃO SOBRE RISCOS ${ }^{1}$
}

\section{THE INFLUENCE OF CORPORATE GOVERNANCE AND AUDIT IN RISK DISCLOSURE}

\author{
Sara Serra \\ Doutora em Contabilidade (Universidade do Minho) \\ Escola Superior de Gestão do Instituto Politécnico do Cávado e do Ave (Barcelos, Portugal) \\ sserra@ipca.pt \\ Kátia Lemos \\ Doutora em Contabilidade (Universidade de Santiago de Compostela) \\ Escola Superior de Gestão do Instituto Politécnico do Cávado e do Ave (Barcelos, Portugal) \\ klemos@ipca.pt
}

\section{RESUMO}

Objetivo: O objetivo deste trabalho é analisar a relação existente entre a divulgação de informação sobre riscos e os mecanismos de governança corporativa e o auditor externo.

Fundamento: A opção sobre divulgar mais ou menos informação sobre determinados riscos está intimamente relacionada com os mecanismos de governança corporativa implementados e com as características do auditor, pois estes irão influenciar a decisão sobre as políticas de divulgação a serem adotados pela entidade.

Método: Foi realizado um ensaio teórico relacionado com o tema em apreço, no sentido de identificar resultados prévios que comprovem a existência da influência da governança corporartiva e do auditor na divulgação sobre riscos.

Resultados: Verifica-se que determinadas características do conselho de administração, a estrutura de propriedade e a existência de um comitê de gestão de risco autônomo, o valor dos honorários recebidos pelo auditor, o tipo de empresa de auditoria, a dimensão da carteira de clientes e a constituição de um comitê de auditoria se encontram associados ao nível e qualidade da divulgação sobre riscos.

Contribuições: Este estudo é inovador pelo fato de centrar-se no papel da governança e do auditor na divulgação de riscos, comprovando a importância destes mecanismos de redução de assimetrias informacional nas políticas de divulgação. Além disso, contribui para a que as empresas reflitam sobre a necessidade de aprimorar estes mecanismos.

Palavras-chave: Divulgação. Riscos financeiros. Auditor externo. Governança corporativa.

\footnotetext{
${ }^{1}$ Artigo recebido em: 23/07/2019. Revisado por pares em: 28/11/2019. Reformulado em: 13/01/2020. Recomendado para publicação: 25/04/2020 por Karla Katiúscia Nóbrega Almeida (Editora Adjunta). Publicado em: 07/09/2020. Organização responsável pelo periódico: UFPB
} 


\section{ABSTRACT}

Objective: The objective of this paper is to analyze the relationship between risk disclosure and corporate governance mechanisms and the external auditor.

Foundation: The option to disclose more or less information about certain risks is closely related to the corporate governance mechanisms implemented and the characteristics of the auditor, as we understand that these bodies will influence the decision on disclosure policies to be adopted by the entity.

Method: For this, a theoretical essay will be carried out, in order to identify previous results that prove the existence of the influence of the corporate governance and the auditor in the disclosure about the risks.

Results: It is noted that certain characteristics associated with the Board of Directors, the ownership structure and the existence of an independent risk management committee, the amount of fees received by the auditor, the type of audit firm to which it is associated, the size of its client portfolio and the establishment of an Audit Committee are associated with the level and quality of risk disclosure.

Contributions: This study is groundbreaking in that it focuses on the role of corporate governance and the auditor in risk disclosure, proving the importance of these mechanisms for reducing information asymmetries in disclosure policies. It also helps companies to reflect on the need to improve these mechanisms.

KEYWORDS: Disclosure. Financial Risks. External Auditor. Corporate Governance.

\section{INTRODUÇÃO}

Os escândalos financeiros protagonizados pela WorldCom e pela Enron, bem como a acusação da empresa de auditoria Arthur Andersen pela maior fraude de auditoria em todo o mundo, abalaram a confiança nas auditorias e no desempenho do auditor. Estes acontecimentos, juntamente com a crise econômica e financeira, fizeram com que a atenção na gestão e na qualidade da divulgação aumentasse (Zango, Kamardin, \& Ishak, 2016).

A crise financeira criou um ambiente de desconfiança na informação divulgada pelas empresas, principalmente no que concerne à divulgação sobre os riscos incorridos, concluindo-se que as informações divulgadas nem sempre deixaram claro o tipo e a magnitude dos riscos (G20, 2009). Por conseguinte, as empresas procuraram soluções para melhorarem os seus relatórios financeiros e uma dessas soluções é a divulgação de informação para os acionistas (Salehi, Moradi, \& Paiydarmanesh, 2017), os quais necessitam de informações confiáveis e completas para tomarem as suas decisões.

A divulgação sobre riscos, que se consubstancia em informação relacionada com a incerteza da entidade e sobre as estratégias adotadas na sua gestão, ajuda os investidores a avaliar os riscos das empresas e a prever o seu valor de mercado (Abdullah, Shukor, \& Rahmat, 2017), reduzindo as assimetrias de informação e aumentando a liquidez dos mercados (Elshandidy \& Neri, 2015). Para as entidades financeiras, a divulgação sobre risco, sobretudo de mercado, é vista até como uma ferramenta para evitar a crise bancária (Al-Hadi, Hasan, \& Habib, 2016).

Todavia, a divulgação de informação está também associada a determinados custos indiretos, difusos e difíceis de medir (para além dos custos diretos de recolher e tratar a informação) (Larrán Jorge \& García-Meca, 20004), que deverão ser considerados na definição das políticas de divulgação a adotar. Assim, algumas das teorias que procuram explicar o processo de decisão sobre que tipo de informação divulgar centram-se na análise dos custos e benefícios da divulgação.

A Teoria da Sinalização, também conhecida por Teoria da Assimetria de Informação, que se desenvolveu a partir dos trabalhos de George Akerlof (1970) e Michael Spence (1973), centra-se na 
assimetria de informação entre os gestores e os detentores de capital e credores da sociedade e entre potenciais investidores. Assume-se que os gestores possuem melhores informações sobre os futuros fluxos de caixa da empresa e os acionistas terão incentivos para induzir os gestores a revelar essa informação ao mercado de capitais, de forma a ajustar o valor da empresa aos futuros fluxos de caixa esperados pelo gestor (Bar-Yosef \& Livnat, 1984). Assim, as empresas de maior qualidade tenderão a optar por políticas de divulgação que lhes permitam revelar a sua qualidade superior, enquanto as empresas de menor qualidade tenderão a esconder a sua qualidade inferior (Morris, 1987). Contudo, uma vez que a não divulgação de determinada informação poderá ser interpretada como um sinal para o mercado, as empresas tendem a optar pela sua divulgação. No limite, teoricamente, atinge-se o equilíbrio com a divulgação total, por parte de todas as empresas, uma vez que todas terão incentivos para divulgar todos os atributos que as distinguem positivamente das demais, de forma a maximizar os seus próprios interesses (Campbell, Shrives, \& Bohmbach-Saager, 2001).

O compromisso de aumentar os níveis de divulgação reduz a possibilidade de assimetrias de informação, quer entre a própria empresa e os seus acionistas, quer entre potenciais compradores e vendedores das ações da empresa, diminuindo a probabilidade de emissão de capital a valores mais baixos (Leuz \& Verrecchia, 2000; Botosan, 1997). De acordo com Verrecchia (1999), maiores níveis de divulgação resultam em mercados mais líquidos e, por isso, em menores custos de capital, o que representa um incentivo à divulgação total.

A Teoria de Proprietary Costs foca no pressuposto de que as empresas limitam a divulgação voluntária de informação devido à existência de custos associados à própria divulgação. Estes custos não estão apenas relacionados com os custos de preparação e disseminação da informação, mas, principalmente, com os custos associados com a divulgação de determinada informação, que possa ser estrategicamente utilizada pelos agentes de mercado em seu próprio benefício e em detrimento da empresa divulgadora.

A decisão sobre a divulgação de informação ao investidor é influenciada pela preocupação de que tal informação possa danificar a posição competitiva de determinada empresa no mercado (Healy \& Palepu, 2001; Larrán Jorge \& García-Meca, 2004). Por exemplo, a divulgação detalhada sobre novos produtos contém informação sobre as perspectivas futuras da empresa para os seus acionistas, mas também pode revelar informação estratégica aos seus competidores. Esta informação contém efeitos positivos e negativos para os acionistas. Os efeitos negativos são os proprietary costs (Darrough, 1993).

Segundo esta teoria, a informação detida por determinada empresa é considerada proprietary quando a decisão de entrar no mesmo mercado, tomada por outra empresa competidora, depende da percepção que detém da informação da primeira. O relatório financeiro publicado pela empresa é entendido como uma fonte de informação para o competidor (Dye, 1985). Assim, determinada empresa limita a informação divulgada ao mercado devido à existência de custos relacionados com a divulgação de informação que pode ser utilizada pelos seus competidores e outras entidades, de forma prejudicial. Os modelos teóricos (Dye, 1985; Verrecchia, 1983; Newman \& Sansing, 1993; Gigler, 1994; Baiman \& Verrecchia, 1996; Hayes \& Lundholm, 1996) sugerem que, ao tomar a decisão sobre a divulgação de determinada informação ao mercado, as empresas terão sempre em consideração que essa informação poderá afetar a sua posição competitiva no mercado.

De acordo com Verrecchia (1983), quanto maiores forem os proprietary costs associados à divulgação de informação, menores serão as reações dos investidores à retenção de informação relevante e, por isso, menor será a probabilidade de divulgação de informação voluntária. Justifica-se, deste modo, porque é que, no equilíbrio, apesar de existirem incentivos para que as empresas aumentem o nível de divulgação como uma forma de redução das assimetrias de informação, não prevalece a divulgação total (Leuz, 2004). 
Por outro lado, de acordo com a Teoria da Legitimidade, a divulgação de informação é utilizada pelas empresas como uma forma de reagir a fatores ambientais (econômicos, políticos e sociais), mas também como uma forma de legitimar as suas ações (Guthrie \& Parker, 1989; Campbell, Moore, \& Shrives 2006). Qualquer falha no cumprimento das atividades esperadas pela sociedade poderá levar ao rompimento do contrato. Deste modo, as empresas precisam divulgar informação suficiente acerca das suas atividades para que a sociedade possa julgar a sua atuação. Através da divulgação, as organizações comunicam a todos os seus stakeholders que estão a cumprir com os termos do contrato social e asseguram, assim, a legitimidade necessária à sua sobrevivência. As empresas podem recorrer aos relatórios anuais e a outras informações institucionais, ou mesmo às suas páginas eletrônicas, como táticas de legitimação, influenciando a percepção que os diversos stakeholders têm da empresa (Lightstone \& Driscoll, 2008).

Segundo a Teoria da Agência, para além da divulgação, alguns mecanismos de governança corporativa das entidades, como determinadas características associadas ao conselho de administração e ao auditor externo têm sido identificados como soluções para aumentar a transparência na relação gestor-investidor, e diminuir a assimetria informacional (Carmona, Fuentes, \& Ruiz, 2016; Kuntari, Chariri, \& Nurdhiana, 2017). Elshandidy e Neri (2015) argumentam que a literatura tem comprovado que a função de monitoração dos mecanismos de governança corporativa tem contribuído para uma melhor divulgação.

Jensen e Meckling (1976) salientam que a auditoria dilui os efeitos adversos da separação entre propriedade e controle. O objetivo passa por aprovar as demonstrações financeiras através de uma investigação imparcial e com conhecimentos técnicos reconhecidos (Bortolon, Neto, \& Santos, 2013). Segundo Sikka, Filling e Liew (2009), os auditores são capazes de mediar a incerteza e construir um relato sobre o negócio da entidade, permitindo aos stakeholders gerir, adequadamente, os riscos. Aliás, a Internacional Standard on Auditing 315 (Identifying and Assessing the Risks of Material Misstatement through Understanding the Entity and Its Environment) preconiza os procedimentos de avaliação de risco para o auditor conhecer a entidade e o seu ambiente e, deste modo, identificar e avaliar os riscos de distorção material devido à fraude ou erro (International Auditing and Assurance Standards Board, 2018).

No que concerne às atividades associadas a algum tipo de risco, faz com que as empresas ponderem, de forma séria, o que deverão e o que não deverão divulgar sobre este tipo de operações. As empresas enfrentam um sério dilema: por um lado, encontram-se tentadas a esconder a sua atuação no mercado, com receio de que a mesma possa ser interpretada como estando associada a elevados riscos; por outro lado, a falta de divulgação, clara e detalhada, sobre este tipo de operações, pode também conduzir a uma má percepção da verdadeira natureza das operações. Além disso, é importante a percepção dos riscos efetivamente incorridos e, principalmente, das práticas adotadas na gestão desses riscos. A falta de divulgação ou a divulgação de informação insuficiente pode levar a uma má percepção da sua atuação, levando a empresa a incorrer em custos de legitimidade e em custos de capital. As empresas sofrem, por isso, pressões, emitidas pelos diversos stakeholders, que as incentivam à divulgação de informação suficiente e confiável.

Face ao exposto, o objetivo deste trabalho é analisar a relação existente entre a divulgação de informação sobre riscos e os mecanismos de governança corporativa e o auditor externo. Por conseguinte, através de um ensaio teórico, pretende-se comprovar que estes mecanismos de redução da assimetria informacional exercem alguma influência na divulgação, recorrendo a uma extensiva revisão de estudos empíricos. Estamos certas de que a opção sobre divulgar mais ou menos informação sobre determinados riscos está intimamente relacionada com os mecanismos de governança implementados pelas empresas e com as características do auditor, pois entendemos que estes órgãos irão influenciar a decisão sobre as políticas de divulgação a serem adotados pela entidade. 
Este trabalho pretende contribuir para a investigação na área da divulgação de informação sobre riscos, sendo inovador no fato de se centrar especificamente no papel da governança das sociedades e no auditor na tomada de decisão sobre a quantidade e qualidade de informação a divulgar.

Deste modo, após esta introdução, na seção seguinte apresentamos uma revisão dos estudos empíricos que se debruçaram sobre a análise da informação divulgada, quer pelas empresas do setor financeiro, quer pelas empresas não financeiras. Nas secções 2 e 3 são analisadas as variáveis associadas à governança corporativa das entidades e ao auditor externo, respectivamente, identificadas como estando associadas à divulgação sobre riscos. Finalmente, são apresentadas as principais conclusões, limitações e sugestões para futuras investigações.

\section{DIVULGAÇÃO DE INFORMAÇÃO SOBRE RISCOS}

A divulgação sobre riscos é entendida como todo tipo de informação, divulgada nas demonstrações financeiras, relacionada com a incerteza, tais como as estimativas e julgamentos dos gestores sobre imparidades, instrumentos financeiros, economia, política, finanças, gestão de riscos e sistemas de controlo interno (Khlif \& Hussainey, 2016). De acordo com Kim e Yasuda (2018), a divulgação sobre riscos deverá conter toda a informação que afete as decisões dos investidores e incluir todos os fatores que influenciem a performance futura da empresa.

Abdullah et al. (2017) definem risco como qualquer "dano e ameaça" ou "oportunidade e perspectiva" que ocorra como resultado de mudanças no ambiente de negócios ou possa ter um impacto na empresa. Neste sentido, a divulgação sobre gestão de riscos compreende dois elementos: o primeiro elemento refere-se a qualquer divulgação relacionada com o risco enfrentado por uma empresa, ou seja, qualquer "oportunidade e perspectiva" ou qualquer "dano e ameaça" que possa ter ocorrido ou afetará a empresa. O segundo elemento refere-se à informação de como cada oportunidade, perspectiva, dano e ameaça é gerido pela empresa.

De acordo com Carmona et al. (2016), o conteúdo informativo dos relatórios financeiros é motivo de grande preocupação, pois os mercados de capitais estão cada vez mais exigentes, e fornecer informações verdadeiras e relevantes torna-se crucial para o seu bom funcionamento. A falta de transparência, a exposição ao risco e as fracas estruturas de governança contribuíram para o crescimento da crise financeira.

Moumen, Othman e Hussainey (2016) definem a divulgação de risco como informativa, na medida em que o relato sobre risco reduz a incerteza sobre os negócios e transmite informações sobre perspectivas futuras para investidores externos. A divulgação sobre risco deve, consequentemente, ajudar os investidores a formar melhores expectativas sobre os ganhos futuros das empresas e a incluir mais informações específicas sobre as empresas nos preços das ações.

Com o objetivo de promover a transparência e aumentar a qualidade da divulgação, reduzindo as assimetrias de informação, os riscos e a gestão de riscos são potencialmente úteis para analistas, investidores e restantes dos stakeholders. Estas divulgações permitem avaliar a capacidade dos gestores para lidarem com a volatilidade dos mercados, com a incerteza, bem como o seu impacto no desempenho da empresa (Dobler, Lajili, \& Zéghal, 2011).

Nesse sentido, vários autores debruçaram-se sobre o estudo da informação sobre risco que é divulgada pelas empresas, quer nas empresas financeiras, quer nas empresas não financeiras, conforme expomos em seguida.

\subsection{Nas entidades financeiras}

Alguns autores, tais como Woods e Marginson (2004), Oliveira, Caires, Silva e Ferreira (2008) e Bischof (2009) preocuparam-se em estudar a informação divulgada, exclusivamente, pelas instituições financeiras. De acordo com Al-Hadi et al. (2016), a divulgação sobre risco, especialmen- 
te sobre risco de mercado, é de extrema importância para as entidades financeiras, uma vez que é vista como uma ferramenta para evitar a crise bancária.

Outros autores preocupam-se em analisar a informação divulgada por aquelas instituições, apenas, no que concerne aos riscos incorridos (Alves \& Cherobim, 2009), fatores determinantes (Linsley, Shrives, \& Crumpton, 2006; Woods, Dowd, \& Humphrey, 2008, Dantas, Rodrigues, Rodrigues, \& Capelletto, 2010) e ao grau de cumprimento das recomendações contidas na publicação conjunta do Basel Committee on Banking Supervision (BCBS) e da International Organization of Securities Commissions (IOSCO) (1999), relativamente à divulgação sobre instrumentos financeiros e riscos associados à negociação dos mesmos (BCBS e IOSCO, 1997, 1999; BCBS, 2003; Xavier, 2003; Yong, Chalmers, \& Faff, 2005; Feria Dominguez \& Oliver Alfonso, 2006; Beneditto \& Silva, 2008; Carneiro, 2008).

Relativamente à informação sobre riscos e aos seus fatores determinantes, Linsley et al. (2006) analisaram a divulgação, pelos maiores bancos do Reino Unido e do Canadá, sobre riscos e políticas de gestão de risco. Por sua vez, Woods et al. (2008) estudaram a divulgação sobre risco de mercado pelos 25 maiores bancos do mundo, através de uma análise de conteúdo dos relatórios anuais relativos aos exercícios de 2000 a 2006. Dhar (2014) analisou a informação divulgada por 19 instituições financeiras indianas, no período de 2012 a 2013, e El-Bannany (2015) analisou a informação sobre risco de crédito divulgada pelos bancos dos Emirados Árabes Unidos, no período de 2006 a 2009. Estes autores identificam como fatores determinantes da divulgação sobre riscos: o tamanho da entidade e o número de definições de risco (Linsley et al., 2006), a extensão do relatório anual (Woods et al., 2008), o nível de rentabilidade (Dhar, 2014; El-Bannany, 2015), o capital estrangeiro, tempo de existência da entidade e investimento em tecnologias de informação (El-Bannany, 2015).

Alves e Cherobim (2009) analisaram o grau de cumprimento das recomendações do Comitê de Basileia sobre risco operacional nos maiores bancos brasileiros e em 9 bancos sediados nos Estados Unidos da América e na Europa. Os resultados comprovam que os bancos sediados no Brasil apresentam menos informação que os bancos situados no estrangeiro, mas que o nível de divulgação aumentou, de 2003 para 2004, em todos os países estudados.

Dantas et al. (2010) avaliaram o impacto do Acordo de Basileia II, na forma como os bancos brasileiros efetuam a divulgação sobre risco de crédito e seus fatores determinantes, no período de 2001 a 2008, enquanto Feria Dominguez e Oliver Alfonso (2006) o fizeram, relativamente ao risco de mercado, para algumas instituições financeiras espanholas, no período de 1999 a 2004 . Os autores concluíram que o nível de divulgação aumentou durante os períodos estudados, mas o grau de cumprimento das recomendações do Comitê de Basileia é, ainda, bastante baixo (Dantas et al., 2010) e a informação divulgada é inconsistente entre entidades, dificultando a comparabilidade e a garantia de transparência, indispensável à salvaguarda da disciplina de mercado (Feria Dominguez \& Oliver Alfonso, 2006)

No que concerne ao grau de cumprimento das recomendações do BCBS e da IOSCO, no Brasil, Xavier (2003) analisou a informação divulgada por parte dos 10 maiores bancos brasileiros, durante os exercícios de 2001 e 2002, procurando aferir o grau de cumprimento das recomendações do acordo de Basileia. Já Beneditto e Silva (2008) analisaram a divulgação de 3 instituições financeiras Brasileiras, entre 2001 e 2007, com o objetivo de comparar as práticas adotadas com as recomendações do Comitê de Basileia e do Banco Central do Brasil. Carneiro (2008) e Dhar (2014) estudaram o grau de cumprimento das exigências de divulgação contidas na Norma Internacional de Relato Financeiro (NIRF) 7, do International Accounting Standards Board, com o Pilar III do Acordo de Basileia II, por parte de 3 grandes instituições financeiras brasileiras (Carneiro, 2008) e de 19 instituições financeiras indianas (Dhar, 2014). Todos os estudos revelaram graus de cumprimento 
com as normas referidas bastante reduzidos, apesar de se verificar um ligeiro aumento ao longo dos períodos analisados (Xavier, 2003; Beneditto \& Silva, 2008; Dhar, 2014)

Yong et al. (2005) analisaram o grau de cumprimento das recomendações do BCBS e da IOSCO, por parte de uma amostra de 146 bancos, sediados nos 10 países da Ásia-Pacífico (Austrália, Hong Kong, Japão, Malásia, Nova Zelândia, Filipinas, Taiwan, Tailândia, Singapura e Coreia do Sul), para o exercício econômico de 2002. Os autores utilizaram um índice de divulgação compreendendo todas as recomendações emitidas pelo BCBS e pela IOSCO. Como resultado, foi obtido um valor médio de divulgação de $35 \%$, indicando que muitas das recomendações não são, ainda, respeitadas pelas instituições analisadas. Além disso, observaram-se diferenças nas práticas de divulgação adotadas nos diferentes países, sendo os níveis mais elevados apresentados por instituições sediadas em Singapura, Hong Kong e Austrália, e os mais reduzidos apresentados por instituições sediadas nas Filipinas. A informação divulgada com maior frequência é a de caráter geral e qualitativo, sendo as informações de caráter quantitativo as menos divulgadas.

Em Portugal, Oliveira, Rodrigues e Craig (2011a) analisaram a informação voluntária e obrigatória divulgada por 190 instituições financeiras, durante o período de 2006. Pinto (2013) estudou o grau de cumprimento das recomendações do Comitê de Basileia, relativamente ao risco de liquidez de 14 instituições financeiras, durante o período de 2007 a 2011. Por sua vez, Amaral (2015) analisou a informação sobre riscos financeiros divulgada pelas 5 maiores instituições financeiras, durante o período de 2006 a 2012.

Oliveira et al. (2011a) concluíram que o nível de divulgação é maior nas entidades que adotaram o normativo do IASB. Todavia, a divulgação sobre riscos é ainda muito escassa, revelando deficiências no nível da transparência da informação financeira. Por sua vez, Pinto (2013) concluiu que as instituições analisadas evidenciam um nível baixo de divulgação e que a dimensão da entidade e o período econômico são fatores determinantes de maiores níveis de divulgação.

Já Amaral (2015) conclui que o nível de divulgação sobre riscos financeiros é crescente ao longo do período analisado, e que se encontra positivamente associado à rentabilidade, à qualidade do crédito, à liquidez, à eficiência operativa e à produtividade dos bancos, e negativamente associado à dimensão e solidez das instituições financeiras.

Mais recentemente, na sequência da crise financeira internacional, Amaral (2015), ElBannany (2015) e Aryani e Hussainey (2017) analisaram a divulgação sobre riscos nas entidades financeiras, procurando aferir a influência das pressões exercidas sobre estas entidades para aumentarem o nível de informação sobre riscos. Todos os autores estudados obtiveram evidência empírica de um aumento no nível de divulgação nos períodos durante e após a crise financeira.

\subsection{Nas entidades não financeiras}

Na sequência da mais recente crise financeira internacional, surgiram alguns estudos que analisaram a informação divulgada pelas instituições não financeiras no que se refere aos riscos financeiros (Lajili, 2009; Dobler et al., 2011; Oliveira, Rodrigues, \& Craig, 2011b; Miihkinen, 2013; Ali \& Taylor, 2014; Elshandidy \& Neri, 2015; Silva, Albuquerque, Marcelino, \& Quirós, 2015; Madrigal, Guzmán, \& Guzmán, 2015; Allini, Rossi, \& Hussainey, 2016; Habtoor, Ahmad, Mohamad, \& Che Haat, 2017; Dey, Hossain, \& Rezae, 2018; Kim \& Yasuda, 2018).

Os resultados destes estudos evidenciaram que as empresas não divulgam informação suficiente e adequada à tomada de decisão dos stakeholders (Ali \& Taylor, 2014), inibindo-os de avaliarem corretamente o perfil de risco das empresas (Scantlebury \& Alleyne, 2015). A informação divulgada é bastante limitada ou vaga (Ali \& Taylor, 2014; Dey et al., 2018), essencialmente de caráter qualitativo, e relativa a eventos passados e presentes (Dobler et al., 2011).

No que se refere ao impacto da obrigatoriedade de adoção de determinadas normas contábeis, que definem requisitos mínimos de divulgação obrigatória sobre riscos, Kim e Yasuda (2018) 
analisaram a informação divulgada pelas empresas cotadas na Bolsa de Tóquio e concluíram que o risco diminuiu após a introdução de divulgação de caráter obrigatório, sugerindo que esta obrigatoriedade reduz o custo de capital. Os autores aferiram, ainda, a existência de uma associação positiva entre o número de itens divulgados e o total de risco, indicando que a divulgação sobre riscos tem um impacto crescente na percepção que os investidores têm sobre o risco das empresas. Por sua vez, Dey et al. (2018) analisaram a informação divulgada por 48 empresas industriais de Bangladesh, no período de 2010 a 2015, e concluíram que, apesar da obrigatoriedade de divulgação definida no normativo contábil, aplicável a partir de 2010, as empresas analisadas ainda não divulgam a informação sobre riscos de forma consistente e uniforme.

No que se refere ao impacto da crise financeira internacional sobre as práticas de divulgação sobre riscos, Miihkinen (2013) analisou a divulgação das empresas não financeiras cotadas na Bolsa de Helsínquia, durante o período de 2006 a 2009, e concluiu que o nível de divulgação aumentou ao longo do período analisado, especialmente no que se refere à informação sobre as estratégias de gestão de risco. Estes resultados são indicadores de que as pressões exercidas sobre as empresas finlandesas para aumentarem os níveis de relato sobre riscos influenciaram os gestores a alterar suas práticas de divulgação.

Elshandidy e Neri (2015) analisaram o impacto da governança corporativa na divulgação voluntária e obrigatória sobre riscos, considerando uma amostra de 290 empresas do Reino Unido e 88 empresas de Itália, durante o período de 2005 a 2010. O estudo permitiu concluir que as empresas italianas divulgam mais informação sobre riscos nos períodos durante e após a crise financeira do que nos períodos que a antecederam.

Em Portugal, Oliveira et al. (2011b) analisaram a informação divulgada por uma amostra de 81 empresas não financeiras (cotadas e não cotadas) durante o período de 2005, enquanto Silva et al. (2015) analisaram a divulgação sobre riscos das empresas não financeiras cotadas na Euronext de Lisboa, nos períodos de 2011 e 2012. Os resultados do estudo de Oliveira et al. (2011b) revelaram que a adoção das normas internacionais, a partir de 2005, não afetou a quantidade e qualidade da divulgação de forma positiva. A informação predominante é de caráter qualitativo, genérica, vaga, referente a eventos passados e inadequada às necessidades dos stakeholders. No mesmo sentido, os resultados do estudo de Silva et al. (2015) demonstram que predomina a informação financeira, de caráter qualitativo, relativa ao passado e classificada como 'boas notícias'.

No que se refere aos fatores determinantes da divulgação sobre riscos, os autores estudados concluíram que o nível de relato está associado ao tamanho da entidade (Lajili, 2009; Oliveira et al., 2011b; Elshandidy \& Neri, 2015; Madrigal et al., 2015; Allini et al., 2016; Habtoor et al., 2017, Dey et al., 2018), ao setor de atividade (Lajili, 2009), ao fato da empresa se encontrar cotada em bolsa (Oliveira et al., 2011b), ao nível de liquidez (Elshandidy \& Neri, 2015; Oliveira et al., 2011b) e de rentabilidade (Elshandidy \& Neri, 2015; Dey et al., 2018), aos níveis de risco (Elshandidy \& Neri, 2015; Madrigal et al., 2015) e ao tipo de auditor (Habtoor et al., 2017).

\section{A INFLUÊNCIA DA GOVERNANÇA CORPORATIVA NA DIVULGAÇÃO SOBRE RIS- COS}

Recentemente surgiu uma linha de investigação que procurou relacionar a divulgação sobre riscos com algumas variáveis associadas à governança corporativa das entidades (Lajili, 2009; Ismail \& Rahman, 2011; Barakat \& Hussainey, 2013; Elshandidy \& Neri, 2015; Carmona et al., 2016; Moumen et al., 2016; Allini et al., 2016; Ashfaq, Zhang, Munaim, \& Razzaq, 2016; Al-Hadi et al., 2016; Abdullah et al., 2017).

A divulgação corporativa pode estar vinculada à estrutura de governança da sociedade, pois é a própria estrutura de governança que incentiva um gestor a adotar as melhores políticas de divulgação. Os efeitos da governança corporativa nas práticas de relato atenuam as assimetrias de 
informação (Carmona et al., 2016) e melhoram a função da gestão. Informações precisas sobre riscos, como mecanismo de controle externo, que reduz os custos de agência, são fundamentais para os acionistas, analistas e investidores, permitindo-lhes avaliar o perfil de risco de uma empresa, estimar o seu valor de mercado e tomar decisões de investimento precisas (Elshandidy \& Neri, 2015).

Como os participantes do mercado não conseguem observar as ações e a credibilidade da administração diretamente, muitas vezes utilizam-se dos mecanismos de governança corporativa para avaliar a qualidade da divulgação sobre riscos das empresas, uma vez que se assume que uma governança saudável se encontra associada à uma eficiente gestão de riscos (Elshandidy \& Neri, 2015).

Na sequência da crise financeira, deficiências nos níveis de governança corporativa e ineficientes sistemas de controle interno e de gestão de risco foram identificados como fatores determinantes do colapso de algumas empresas (Ismail \& Rahman, 2011).

Deste modo, têm sido identificados como fatores determinantes da divulgação sobre riscos determinadas características associadas ao conselho de administração, a existência de um comitê de gestão de risco autônomo, a estrutura de capital da sociedade, entre outros, conforme expomos nas seções seguintes.

\subsection{Características do conselho de administração}

\subsubsection{Tamanho do conselho de administração}

De acordo com Elshandidy e Neri (2015), o tamanho do conselho de administração é um elemento-chave para determinar a sua eficácia. Como tal, argumenta-se que um conselho de administração pequeno não possui conhecimentos suficientes e pode sofrer com o domínio do diretor executivo (CEO). Por outro lado, os grandes conselhos desfrutam de vasta experiência e conhecimentos mais diversificados, o que resulta numa monitoração mais efetiva (Moumen et al., 2016). Por conseguinte, os resultados dos estudos de Elshandidy e Neri (2015) e Moumen et al. (2016) evidenciam que maiores conselhos de administração contribuem para o aumento da divulgação sobre riscos.

\subsubsection{Nível de qualificação dos membros do conselho de administração}

Um conselho de administração eficaz deve ser composto por indivíduos que tenham conhecimento sobre os procedimentos contábeis, para garantir o fornecimento de boa divulgação aos acionistas e ao público. Se o conselho de administração possuir indivíduos com formação acadêmica em contabilidade e finanças, estes irão divulgar mais informações para melhorar a imagem corporativa da empresa e a credibilidade da equipe de gestão (Ismail \& Rahman, 2011).

Assim, Ismail e Rahman (2011) concluíram que a qualificação dos membros do conselho de administração influencia positivamente o nível de divulgação sobre risco. Todavia, Allini et al. (2016) obtiveram evidência empírica de uma associação negativa entre o nível de qualificações dos membros do conselho de administração e o nível de divulgação sobre riscos.

\subsubsection{Independência dos membros do conselho de administração}

De acordo com a Teoria da Agência, o papel dos diretores não executivos independentes é crucial para equilibrar o conselho de administração e monitorar os administradores da empresa (Ismail \& Rahman, 2011; Barakat \& Hussainey, 2013). Assim, é mais provável que um maior número de diretores independentes resulte em níveis mais elevados de transparência (Barakat \& Hussainey, 2013; Moumen et al., 2016).

Os diretores independentes podem ter mais incentivos para aumentar os níveis de divulgação voluntária e, assim, sinalizar a sua falta de cumplicidade com os líderes, gestores e os acionis- 
tas majoritários e a sua própria capacidade de melhorar o valor de mercado da empresa (Elshandidy \& Neri, 2015).

Os diretores internos não têm incentivos suficientes no que diz respeito à qualidade da divulgação, por causa do seu papel na gestão da empresa e porque o seu comportamento pode estar sujeito a um maior escrutínio. Por outro lado, espera-se que os diretores não executivos prestem consultoria independente aos diretores executivos. Acredita-se que estes desempenham um papel crucial na monitoração do desempenho dos gestores e na limitação de seu oportunismo, o que pode levar à redução dos conflitos de agência entre gerentes e proprietários (Moumen et al., 2016).

Lajili (2009), Oliveira et al. (2011b), Barakat e Hussainey (2013), Elshandidy e Neri (2015), Carmona et al. (2016), Moumen et al. (2016) e Ashfaq et al. (2016) obtiveram evidência empírica da existência de uma associação positiva entre o nível de divulgação sobre riscos e o número de diretores independentes no conselho de administração. Todavia, o estudo de Ismail e Rahman (2011) revelou a inexistência de qualquer associação entre a divulgação sobre riscos e a existência de diretores não executivos independentes.

\subsection{Concentração de capital}

A estrutura de propriedade influencia o nível de informação divulgada, uma vez que, quanto maior for a concentração de capital, menor será a probabilidade de divulgação de informação para o exterior. De fato, de acordo com a Teoria da Agência, a separação entre a propriedade e o controle das empresas aumenta a probabilidade de conflitos entre as partes e, por isso, gera maiores custos de monitoração (Fama \& Jensen, 1983b). Deste modo, existirão maiores incentivos à divulgação quando a separação entre os detentores de capital e os gestores for maior, ou seja, quando a concentração de capital é menor.

O estudo de Carmona et al. (2016) revelou a existência de uma associação positiva entre o nível de divulgação sobre riscos e a concentração de capital. Contrariamente, o estudo de Elshandidy e Neri (2015) não evidenciou qualquer associação entre a concentração de capital e a divulgação sobre riscos, quer nas empresas do Reino Unido, quer nas empresas italianas.

\subsection{Comitê de gestão de risco}

Tradicionalmente, a supervisão dos riscos encontra-se sobre a responsabilidade da comissão de auditoria. Todavia, na sequência da mais recente crise financeira internacional, muitas empresas, especialmente as entidades financeiras, têm optado pela criação de um comitê de gestão de risco autônomo (Al-Hadi et al., 2016).

De acordo com Al-Hadi et al. (2016), uma vez que o comitê de gestão de risco se concentra e especializa em monitorar e gerir o risco, a sua existência fortalece o sistema de gestão de risco das empresas, o que eventualmente leva a uma melhoria na divulgação sobre risco.

Tal como no conselho de administração, o desempenho do comitê de gestão de risco também depende da sua composição. Assim, entende-se que os comitês de gestão de risco com maior número de diretores independentes e cujos membros possuem mais habilitações ou experiência nas áreas de finanças e contabilidade revelaram melhor desempenho na gestão de risco e na qualidade do relato sobre riscos (Al-Hadi et al., 2016). Deste modo, Al-Hadi et al. (2016) e Abdullah et al. (2017) obtiveram evidência empírica de que as empresas que possuem um comitê de gestão de risco autônomo divulgam mais informação sobre riscos. Por outro lado, o tamanho e as qualificações do comitê de gestão de risco estão positivamente associados ao nível de divulgação (Al-Hadi et al., 2016). 


\section{O AUDITOR COMO FATOR DETERMINANTE DA DIVULGAÇÃO SOBRE RISCOS}

A auditoria desempenha um papel importante no relato financeiro (Rijwani \& Rajpurohit, 2017 e Nwanyanwu, 2017), dado que visa garantir que as demonstrações financeiras comunicam informações relevantes e confiáveis para os stakeholders. O'Reilly (2010) acrescenta que o relatório resultante da auditoria desempenha um papel crucial no alerta do risco, essencialmente de falência da entidade auditada, bem como na sua previsão e explicação, permitindo que os stakeholders tomem decisões de alocação de capital mais informadas e adequadas.

De acordo com Fukukawa e Kim (2017), os auditores, como estão preocupados com a qualidade de sua auditoria, tentam influenciar as práticas de divulgação de riscos de negócios dos clientes. Dado que a administração tem algum poder discricionário sobre o relato de riscos de negócios, os auditores podem obter uma vantagem competitiva ao fornecer conselhos úteis aos gerentes em relação às informações que devem ser divulgadas e à extensão e tipo de divulgação que é apropriado. Nesta linha de pensamento, Barako, Hancock e Izan, (2006) relatam que a auditoria pode ter um efeito marcante no grau de informação voluntária divulgada nos relatórios anuais da empresa. Sobre esse propósito, Barako et al. (2006) afirmam que, embora seja de inteira responsabilidade da administração preparar os relatórios anuais, uma empresa de auditoria externa pode influenciar significativamente a quantidade de informações divulgada. Nesta linha de pensamento, Elshandidy e Neri (2015) afirmam que a qualidade da auditoria influencia significativamente a divulgação de risco das empresas. Assim, Sikka et al. (2009) afirmam que os auditores se revelam capazes de mediar a incerteza e construir um relato objetivo de assuntos referentes ao negócio da entidade, permitindo aos stakeholders gerir adequadamente os riscos.

A auditoria está relacionada com o nível de divulgação e qualidade da informação financeira, todavia esta influência depende de algumas variáveis que passamos a apresentar, pois, tal como afirmam Nelson e Tay (2005), os atributos do auditor afetam o desempenho da auditoria.

\subsection{Honorários do auditor}

O processo de auditoria é afetado pela quantidade de honorários recebida pelo auditor. Os estudos de Babatolu, Aigienohuwa e Uniamikogbo (2016), Kuntari et al. (2017) e Abdul-Rahman, Benjamin e Olayinka (2017) revelaram que os honorários recebidos pelo auditor têm um efeito positivo significativo na qualidade da auditoria.

Face ao exposto, e tendo por base a Teoria da Agência, Adznan e Nelson (2014) concluíram que existe uma relação entre o nível de honorários do auditor e o nível de divulgação sobre instrumentos derivativos. De forma semelhante, Yang, Yu, Liu e Wu (2018) aferiram que os honorários de auditoria estão significativa e positivamente relacionados com a divulgação dos riscos financeiros, estratégicos e operacionais da entidade auditada.

\subsection{Tipo de empresa de auditoria}

Vários estudos empíricos têm demonstrado que o tipo de empresa de auditoria influencia o processo de auditoria, sendo este de maior qualidade nas empresas de auditoria multinacionais, atualmente designadas de Big Four (Aronmwan, Ashafoke, \& Mgbame, 2013; Pham, Duong, Pham, \& Ho, 2017; Jiang, Wang, \& Wang, 2018 e Asthana, Kalelkar, \& Raman, 2018). Esta constatação deve-se ao receio da perda de reputação e custos daí resultantes (DeAngelo, 1981; Myers, Myers, \& Omer, 2003), mas, também, ao fato de se presumir que as Big Four possuem maior competência técnica (DeAngelo, 1981).

Apoiado nas premissas anteriores, os estudos empíricos de Oliveira et al. (2011), Mokhtar e Mellett (2013), Campbell, Chen, Dhaliwal, Lu e Steele (2014), Carmona et al. (2016), Zango, et al. (2016) e Fukukawa e Kim (2017), Habtoor et al. (2017), bem como Dey et al. (2018) revelaram uma associação positiva entre a divulgação de riscos e o tipo de empresa de auditoria. De fato, empre- 
sas auditadas pelas Big Four tendem a divulgar mais informações sobre risco. Lennox (1999) afirma, ainda, que as Big Four exigem que os seus clientes divulguem mais informações sobre risco devido ao maior risco de litígio.

\subsection{Dimensão da carteira de clientes do auditor}

De acordo com Gómez e Ruiz (2000), a dimensão da carteira de clientes do auditor está relacionada com o processo de auditoria, pois, segundo DeAngelo (1981), existe menor probabilidade destes se deixarem pressionar por um cliente, sendo, portanto, mais independentes. A razão para esta ocorrência está na dependência econômica que uma escassa carteira de clientes provoca, motivando a continuação da relação contratual, podendo levar o auditor a agir de forma oportunista, de acordo com os interesses do auditado.

A independência das empresas de auditoria com maior carteira de clientes permite que estas influenciem os relatórios financeiros das empresas auditadas, com o intuito de satisfazer as necessidades dos stakeholders, já que a sua reputação como auditores, em parte, depende de como os stakeholders percebem o resultado do seu trabalho (Barako et al., 2006).

De acordo com Fukukawa e Kim (2017), a dimensão da carteira de clientes também tem efeito no processo de auditoria, dado que os auditores com maior número de clientes tendem a possuir maior experiência e maior nível de conhecimento. Com base nesta premissa, os autores concluíram que as empresas auditadas por auditores que possuem um número maior de clientes divulgam mais informações sobre riscos de negócios.

\subsection{Comissão de auditoria}

A ocorrência de inúmeros escândalos financeiros tem levado os stakeholders a reivindicar informação financeira mais transparente e fidedigna. Uma das medidas encontradas para resolver este problema, bem como para promover a independência do auditor e a credibilidade das demonstrações financeiras, foi a criação de comissões de auditoria (em alguns normativos, este órgão é denominado de comitê de auditoria. No entanto, utilizaremos a designação de comissão de auditoria, dado que é a mais usual no normativo português). Em Portugal, este mecanismo de controle foi introduzido em 1999, por intermédio das Recomendações da Comissão do Mercado de Valores Mobiliários sobre o Governo das Sociedades Cotadas.

A comissão de auditoria é um órgão da entidade, criado pelo seu conselho de administração, cuja função é assegurar a integridade da informação financeira, bem como garantir a sua confiabilidade e transparência. Por conseguinte, a comissão de auditoria é vista como um meio de promover práticas capazes de aumentar a capacidade da gestão de cumprir as suas responsabilidades legais, e garantir a credibilidade e objetividade dos relatórios financeiros (Santos, Inácio, \& Vieira, 2015).

A constituição da comissão de auditoria influencia o funcionamento da mesma (Menon \& Williams, 1994), sobretudo o seu nível de independência e competência. Aliás, os estudos empíricos de Goodwin (2003) e Al-Mudhaki e Joshi (2004) aferiram que o funcionamento das comissões de auditoria é mais eficaz quando os seus membros possuem conhecimentos em matérias contábeis e afins.

Além disso, a independência dos membros da comissão de auditoria está associada a uma maior qualidade do relatório financeiro (Carcello, Hollingsworth, \& Neal, 2006). Fama e Jensen (1983 a) afirmam, ainda, que comissões de auditoria eficazes melhoram a qualidade e a credibilidade das demonstrações financeiras, auxiliam o trabalho do conselho de administração e salvaguardam os interesses dos acionistas (Santos et al., 2015).

Na senda deste pensamento, Barako et al. (2006) mencionam que a presença de uma comissão de auditoria está associada ao nível de divulgação de informação financeira. Hernández e Trejo 
(2016) acrescentam que a comissão de auditoria permite aos conselhos de administração monitorar a gestão dos negócios e gerar informações tempestivas relacionadas aos principais eventos de riscos.

Face ao exposto, Mangena e Tauringana (2007), Zhang, Taylor, Qu e Wise (2013) e Carmona et al. (2016) concluíram que existe uma relação significativa e positiva entre a independência da comissão de auditoria e a divulgação de riscos. Corroborando o que foi referido anteriormente, à propósito da competência da comissão de auditoria, Magena e Tauringana (2007) e Hoitash, Hoitash e Bedard (2009) afirmam que existe uma relação positiva entre o grau de conhecimentos dos membros da comissão de auditoria e a divulgação de informação financeira.

Em linha com as investigações anteriores, o estudo de Abdullah et al. (2017) permitiu concluir que a existência de uma comissão de auditoria contribui para uma maior divulgação sobre riscos. A frequência de reuniões deste órgão está, também, positivamente associada à quantidade de informação divulgada, indicando que quanto maior a frequência de reuniões, mais ativa será esta comissão no processo de comunicação de informação relacionada com riscos. Estes resultados foram, também, obtidos por Suárez, García, Méndez e Gutiérrez (2013) e Barakat e Hussainey (2013).

\section{CONSIDERAÇÕES FINAIS, LIMITAÇÕES E SUGESTÕES PARA FUTURAS INVESTIGA- ÇÕES}

O objetivo deste trabalho foi analisar a relação existente entre a divulgação de informação sobre riscos e os mecanismos de governança corporativa das entidades e o auditor externo.

Vários autores têm se debruçado sobre a análise da informação divulgada sobre riscos, quer em entidades do setor financeiro, quer em entidades do setor não financeiro, tendo alguns desses estudos sido realizados em Portugal. A generalidade dos autores conclui que, apesar do aumento das exigências de divulgação sobre riscos nos normativos contábeis aplicáveis e das pressões para o aumento da divulgação, especialmente na sequência da mais recente crise financeira internacional, a maioria das empresas não divulga informação suficiente e confiável para a tomada de decisões dos seus stakeholders. A informação divulgada é incipiente, muito vaga, essencialmente de caráter qualitativo e baseada em acontecimentos passados e presentes, não existindo informação suficiente para aferir sobre o perfil de risco das empresas e suas estratégias de gestão de riscos.

No que se refere à influência dos mecanismos de governança corporativa das entidades sobre a divulgação de riscos, os diversos autores estudados comprovaram que determinadas características associadas ao conselho de administração (tamanho, nível de habilitações dos seus membros e número de diretores independentes), a estrutura de propriedade e a existência de um comitê de gestão de risco autônomo influenciam positivamente a quantidade e qualidade da informação divulgada.

No que se refere ao efeito do auditor externo, os estudos analisados comprovam que o valor dos honorários recebidos pelo auditor, o tipo de empresa de auditoria a que se encontra associado (Big Four/não Big Four), a dimensão da sua carteira de clientes e a composição da comissão de auditoria se encontram associados ao nível e qualidade da divulgação sobre riscos.

Face ao exposto, concluímos que, de fato, tal como esperávamos, se comprova a influência, quer dos mecanismos de governança das sociedades, quer do auditor, na divulgação sobre riscos. A opção por determinada composição do conselho de administração (em termos de tamanho, qualificações e número de diretores independentes), a própria estrutura de propriedade e a decisão sobre a criação de um comitê de gestão de risco estão intimamente associadas às políticas de divulgação sobre riscos que a empresa persegue. Estes órgãos internos influenciam positivamente a quantidade e qualidade de informação divulgada. 
Por outro lado, a escolha do auditor externo também terá impacto na informação sobre riscos que é apresentada aos diferentes stakeholders. Os auditores com maiores honorários, associados a uma das Big Four e com maiores carteiras de clientes estão também preocupados em manter a sua reputação, e, por isso, irão influenciar as entidades auditadas a melhorar os níveis e qualidade da informação. Além disso, a existência de uma comissão de auditoria, composta por maior número de membros independentes e com melhores qualificações influencia positivamente a divulgação de informação sobre riscos.

Este trabalho apresenta, todavia, como limitação, o fato de não se basear em nenhuma observação empírica relativamente ao relato sobre riscos e sobre os efeitos dos mecanismos de governança e do auditor externo na quantidade e qualidade da informação divulgada. Nesse sentido, sugerimos, para futuras investigações, realizar uma análise das informações divulgadas pelas maiores empresas portuguesas, financeiras e não financeiras, procurando aferir sobre as práticas de divulgação de risco adotadas, e sobre a influência do governo das sociedades e do auditor externo nessas práticas de divulgação.

\section{REFERÊNCIAS}

Abdul-Rahman, O., Benjamin, A., \& Olayinka, O. (2017). Effect of audit fees on audit quality: evidence from cement manufacturing companies in Nigeria. European Journal of Accounting, Auditing and Finance Research, 5(1), 6-17.

Abdullah, M., Shukor, Z., \& Rahmat, M. (2017). The influence of risk management committee and audit committee towards voluntary risk management disclosure. Jurnal Pengurusan, 50, 1-20.

Adznan, S., \& Nelson, S. (2014). Financial instruments disclosure practices: evidence from Malaysian listed firms. Procedia-Social and Behavioral Sciences, 164, 62-67.

Akerlof, G. A. (1970). The market for "lemons": quality uncertainty and the market mechanism. The Quarterly Journal of Economics, 84(3), 488-500.

Al-Hadi, A., Hasan, M., \& Habib, A. (2016). Risk committee, firm life cycle, and market risk disclosures. Corporate Governance: An International Review, 24(2), 145-170.

Al-Mudhaki, J., \& Joshi, P. (2004). The role and functions of audit committees in the Indian corporate governance: empirical findings. International Journal of Auditing, 8, 33-47.

Ali, M., \& Taylor, D. (2014). Content analysis of corporate risk in Malaysia. $4^{\text {th }}$ Annual International Conference on Accounting and Finance, 15-24.

Allini, A., Rossi, F., \& Hussainey, K. (2016). The board's role in risk disclosure: an exploratory study of Italian listed state-owned enterprises. Public Money \& Management, fevereiro, 113-120.

Alves, C., \& Cherobim, A. (2009). Análise do nível de divulgação do risco operacional segundo recomendações do Comité de Basileia: estudo em bancos do país e exterior. Revista de Administração MacKenzie, 10(2), 57-86.

Amaral, M. (2015). Divulgação de informação sobre riscos financeiros: evidência empírica no setor bancário português. Dissertação de Mestrado em Banca e Seguros, Instituto Politécnico do Cávado e do Ave.

Aronmwan, E., Ashafoke, T., \& Mgbame, C. (2013). Audit firm reputation and audit quality. European Journal of Business and Management, 5(7), 66-75.

Aryani, D., \& Hussainey, K. (2017). The determinants of risk disclosure in the Indonesian nonlisted banks. Int. J. Trade and Global Markets, 10(1), 58-66.

Ashfaq, K., Zhang, R., Munaim, A., \& Razzaq, N. (2016). An investigation into the determinants of risk disclosure in banks: evidence from financial sector of Pakistan, International Journal of Economics and Financial Issues, 6(3), 1049-1058. 
Asthana, S., Kalelkar, R., \& Raman, K. (2018). Unintended consequences of Big 4 auditor officelevel industry specialisation. International Journal of Accounting, Auditing and Performance Evaluation, 14(2-3), 254-289.

Babatolu, A., Aigienohuwa, O., \& Uniamikogbo, E. (2016). Auditor's independence and audit quality: a study of selected deposit money banks in Nigeria. International Journal of Finance and Accounting, 5(1), 13-21.

Baiman, S., \& Verrecchia, R. E. (1996). The relation among capital markets, financial disclosure, production efficiency and insider trading. Journal of Accounting Research, 34(1), 1-22.

Bar-Yosef, S., \& Livnat, J. (1984). Auditor selection: an incentive-signalling approach. Accounting and Business Research, 14(56), 301-309.

Barakat, A., \& Hussainey, K. (2013). Bank governance, regulation, supervision, and risk reporting: evidence from operational risk disclosures in European banks. International Review of Financial Analysis, 30, 254-273;

Barako, D., Hancock, P., \& Izan, H. (2006). Factors influencing voluntary corporate disclosure by Kenyan companies. Corporate Governance: An International Review, 14(2), 107-125.

Basel Committee on Banking Supervision (2003). Public disclosures by banks: results of the 2001 disclosure survey, BCBS.

Basel Committee on Banking Supervision \& Technical Committee of the International Organization of Securities Commissions (1997). Survey of disclosures about trading derivatives activities of banks and securities firms 1996, BCBS \& IOSCO.

Basel Committee on Banking Supervision \& Technical Committee of the International Organization of Securities Commissions (1999). Trading and derivatives disclosures of banks and securities firms - results of the survey of public disclosures in 1998 annual reports, BCBS \& IOSCO.

Beneditto, E., \& Silva, R. (2008). Análise do nível de transparência das instituições financeiras brasileiras em relação ao Acordo com o Novo Acordo de Capitais (Basileia II) - Um estudo de caso múltiplo. Brazilian Business Review, 5(3), 192-208.

Bischof, J. (2009). The effects of IFRS 7 adoption on bank disclosure in Europe. Accounting in Europe, 6(2), 167-194.

Bortolon, P., Neto, A., \& Santos, T. (2013). Audit costs and corporate governance. Revista Contabilidade e Finanças, 24(61), 27-36.

Botosan, C. A. (1997). Disclosure level and the cost of equity capital. The Accounting Review, 72(3), 323-349.

Breesch, D., \& Branson, J. (2009). The effects of auditor gender on audit quality. The IUP Journal of Accounting Research $\mathcal{E}$ Audit Practices, 8(3-4), 78-107.

Campbell, D., Moore, G., \& Shrives, P. (2006). Cross-sectional effects in community disclosure. Accounting, Auditing $\mathcal{E}$ Accountability Journal, 19(1), 96-114.

Campbell, D., Shrives, P., \& Bohmbach-Saager. H. (2001). Voluntary disclosure of mission statements in corporate annual reports: signaling what and to whom? Business and Society Review, 106(1), 65-87.

Campbell, J., Chen, H., Dhaliwal, D., Lu, H., \& Steele, L. (2014). The information content of mandatory risk factor disclosures in corporate filings. Review of Accounting Studies, 19(1), 396-455.

Carcello, J., Hollingsworth, C., \& Neal, T. (2006). Audit committee financial experts: a closer examination using firm designations. Accounting Horizons, 20(4), 351-373.

Carmona, P., Fuentes, C. D., \& Ruiz, C. (2016). Risk disclosure analysis in the corporate governance annual report using fuzzy-set qualitative comparative analysis. Revista de Administração de Empresas, 56(3), 342-352.

Carneiro, R. (2008). Divulgação de informações sobre instrumentos financeiros e riscos bancários: uma análise comparativa. Dissertação de Mestrado em Administração e Contabilidade, Universidade de São Paulo. 
Dantas, J., Rodrigues, F., Rodrigues, J., \& Capelletto, L. (2010). Determinantes do grau de evidenciação de risco de crédito pelos bancos brasileiros. Revista de Contabilidade \& Finanças, USP, São Paulo, 21(52), 1-27.

Darrough, M. N. (1993). Disclosure policy and competition: Cournot vs. Bertrand. The Accounting Review, 68(3), 534-561.

DeAngelo, L. (1981). Auditor size and audit quality. Journal of Accounting and Economics, 3, 183-199.

Dey, R., Hossain, S., \& Rezae, Z. (2018). Financial risk disclosure and financial attributes among publicly traded manufacturing companies: evidence from Bangladesh. Journal of Risk and Financial Management, 11(50), 1-16.

Dhar, P. (2014). Risk disclosure by select Indian banks with reference to IFRS 7 / IND-AS 32: a study. International Journal of Research in Commerce \& Management, 5(5), 29-37.

Dobler, M., Lajili, K., \& Zéghal, D. (2011). Attributes of corporate risk disclosure: an international investigation in the manufacturing sector. Journal of International Accounting Research, 10(2), 122.

Dye, R. A. (1985). Strategic accounting choice and the effects of alternative financial reporting requirements. Journal of Accounting Research, 23(2), 544-574.

El-Bannany, M. (2015). Global financial crisis and credit risk disclosure in the UAE banks. Risk Governance \& Control: Financial Markets \& Institutions, 5(1), 20-26.

Elshandidy, T., \& Neri, L. (2015). Corporate governance, risk disclosure practices, and market liquidity: comparative evidence from the UK and Italy. Corporate Governance: An International Review, 23(4), 331-356.

Fama, E., \& Jensen, M. (1983a). Agency problems and residual claims. Journal of Law and Economics. 26(2), 327-349.

Fama, E., \& Jensen, M. (1983b). Separation of ownership and control. Journal of Law and Economics, $26(2), 301-325$.

Feria Domínguez, J., \& Oliver Alfonso, M. (2006). Análisis en el marco de BASILEIA II del tratamiento del riesgo de mercado en los informes anuales bancarios. Técnica Contable, 685, 25-33.

Fukukawa, H., \& Kim, H. (2017). Effects of audit partners on clients' business risk disclosure. Accounting and Business Research, 47(7), 780-809.

G20 Working Group (2009). Enhancing sound regulation and strengthening transparency: final report. Documento disponível em http://www.gfintegrity.org/storage/gfip/documents/g20\%20working\%20group\%201\%20report .pdf.

Gigler, F. (1994). Self-enforcing voluntary disclosures. Journal of Accounting Research, 32(2), 224-240.

Gómez, N., \& Ruiz, E. (2000). Un estudio empírico sobre la relación entre informe de auditoría y cambio de auditor. Revista Española de Financiación y Contabilidad, XXIX (105), 705-741.

Goodwin, J. (2003). The relationship between the audit committee and the internal audit function: evidence from Australia and New Zealand. International Journal of Auditing, 7, 263-278.

Guthrie, J., \& Parker, L. D. (1989). Corporate social reporting: a rebuttal of legitimacy theory. Accounting and Business Research, 19(76), 343-352.

Habtoor, O., Ahmad, N., Mohamad, N., \& Che Haat, M. (2017). Linking corporate risk disclosure practices with firm-specific characteristics in Saudi Arabia. Gadjah Mada International Journal of Business, 19(3), 247.

Hayes, R. M., \& Lundholm, R. (1996). Segment reporting to the capital market in the presence of a competitor. Journal of Accounting Research, 34(2), 261-279.

Healy, P. M., \& Palepu, K. G. (2001). Information asymmetry, corporate disclosure, and the capital markets: a review of the empirical disclosure literature. Journal of Accounting and Economics, 31, 405-440. 
Hernández, R., \& Trejo, X. (2016). Risk disclosure: first overview in the Mexican context. World of Accounting Science, 18, 511-536.

Hoitash, U., Hoitash, R., \& Bedard, J. (2009). Corporate governance and internal control over financial reporting: a comparison of regulatory regimes. The Accounting Review, 84(3), 839-867.

International Auditing and Assurance Standards Board. (2018). Handbook of international quality control, auditing, review, other assurance, and related services pronouncements: International Standard on Auditing. 278-333.

Ismail, R. \& Rahman, A. (2011). Institutional investors and board of directors' monitoring role on risk management disclosure level in Malaysia. The IUP Journal of Corporate Governance, 10(2), 37-61.

Jensen, M., \& Meckling, W. (1976). Theory of the firm: managerial behavior, agency costs and ownership structure. Journal of Financial Economics, 3(4), 305-360.

Jiang, J., Wang, I., \& Wang, K. (2018). Big N auditors and audit quality: new evidence from quasiexperiments. The Accounting Review. In-Press.

Khlif, H. \& Hussainey, K. (2016). The association between risk disclosure and firm characteristics: a meta-analysis. Journal of Risk Research, 19(2), 181-211.

Kim, H. \& Yasuda, Y. (2018). Business risk disclosure and firm risk: evidence from Japan. Research in International Business and Finance, 45, 413-426.

Kuntari, Y., Chariri, A., \& Nurdhiana, N. (2017). The effect of auditor ethics, auditor experience, audit fees and auditor motivation on audit quality. Sriwijaya International Journal of Dynamic Economics and Business, 1(2), 203-218.

Lajili, K. (2009). Corporate risk disclosure and corporate governance. Journal of Risk and Financial Management, 2, 94-117.

Larrán Jorge, M., \& García-Meca, E. (2004). Costes, beneficios y factores ligados a la política de divulgación de información financiera. Revista de Contabilidad, 17(14), 75-111.

Lennox, C. (1999). Non-audit fees, disclosure and audit quality. European Accounting Review, 8(2), 239-252.

Leuz, C., \& Verrechia, R. E. (2000). The economic consequences of increased disclosure. Journal of Accounting Research, 38(supplement), 91-124.

Lightstone, K., \& Driscoll, C. (2008). Disclosing elements of disclosure. A test of legitimacy theory and company ethics. Canadian Journal of Administrative Sciences, 25(1), 7-21.

Linsley, P., Shrives, P., \& Crumpton, M. (2006). Risk disclosure: an exploratory study of UK and Canada banks. Journal of Banking Regulation, 7(3/4), 268-282.

Madrigal, M., Guzmán, B., \& Guzmán, C. (2015). Determinants of corporate risk disclosure in large Spanish companies: a snapshot. Contaduría y Administración, 60, 757-775.

Mangena, M., \& Tauringana, V. (2007). Disclosure, corporate governance and foreign share ownership on the Zimbabwe stock exchange. Journal of International Financial Management and Accounting, 18(2), 53-85.

Menon, K., \& Williams, J. (1994). The use of audit committees for monitoring. Journal of Accounting and Public Policy, 13(2), 121-139.

Miihkinen, A. (2013). The usefulness of firm risk disclosures under different firm riskiness, investor-interest, and market conditions: new evidence from Finland. Advances in Accounting incorporating Advances in International Accounting, 29, 312-331.

Mokhtar, E., \& Mellett, H. (2013). Competition, corporate governance, ownership structure and risk reporting. Managerial Auditing Journal, 28(9), 838-865.

Morris, R. D. (1987). Signalling, agency theory and accounting policy choice. Accounting and Business Research, 18(69), 47-56. 
Moumen, N., Othman, H., \& Hussainey, K. (2016). Board structure and the informativeness of risk disclosure: evidence from MENA emerging markets. Advances in Accounting incorporating Advances in International Accounting, 35, 82-97.

Myers, J., Myers, L., \& Omer, T. (2003). Exploring the term of the auditor-client relationship and the quality of earnings: A case for mandatory auditor rotation?. The Accounting Review, 78(3), 779-799.

Nelson, M., \& Tan, H. (2005). Judgment and decision making research in auditing: a task, person, and interpersonal interaction perspective. Auditing: A Journal of Practice $\mathcal{E}$ Theory, 24(Supplement), 41-71.

Newman, P., \& Sansing, R. (1993). Disclosure policies with multiple users. Journal of Accounting Research, 31(1), 92-112.

Nwanyanwu, L. (2017). Audit quality practices and financial reporting in Nigeria. International Journal of Academic Research in Accounting, Finance and Management Sciences, 7(2), 145-155.

O'Reilly, D. (2010). Do investors perceive the going-concern opinion as useful for pricing stocks?. Managerial Auditing Journal, 25(1), 4-16.

Oliveira, A., Caires, N., Silva, O., \& Ferreira, R. (2008). Derivativos: evolução da aplicação das normas contábeis e de evidenciação das demonstrações financeiras mediante as mudanças de normas contábeis no Brasil. Comunicação apresentada no XII Congresso de Contabilidade e Auditoria.

Oliveira, J., Rodrigues, L. \& Craig, R. (2011a). Risk-related disclosure practices in the annual reports of Portuguese credit institutions: an exploratory study. Journal of Banking Regulation, 12(2), 100-118.

Oliveira, J., Rodrigues, L. \& Craig, R. (2011b). Risk-related disclosure by non-finance companies: Portuguese practices and disclosure characteristics. Managerial Auditing Journal, 26(9), 817-839.

Pham, N., Duong, H., Pham, T., \& Ho, N. (2017). Audit firm size, audit fee, audit reputation and audit quality: the case of listed companies in Vietnam. Asian Journal of Finance $\mathcal{E}$ Accounting, 9(1), 429-447.

Pinto, C. (2013). Fatores determinantes da divulgação de informação sobre risco de liquidez: evidencia empírica no sistema financiero português. Dissertação de Mestrado em Contabilidade e Finanças, Instituto Politécnico do Cávado e do Ave.

Rijwani, P., \& Rajpurohit, P. (2017). Assessment of audit quality: an investigation into proxies. Asian Journal of Management, 8(2), 262-266.

Salehi, M., Moradi, M., \& Paiydarmanesh, N. (2017). The effect of corporate governance and audit quality on disclosure quality: evidence from Tehran stock exchange. Periodica Polytechnica Social and Management Sciences, 25, 32-48.

Santos, M., Inácio, H., \& Vieira, E. (2015). Governo das sociedades e a opinião do auditor: evidência portuguesa. Revista Universo Contábil. 11(3), 150-168.

Scantlebury, K., \& Alleyne, P. (2015). A content analysis of risk management disclosures in Barbadian public limited companies' annual reports. Journal of Eastern Caribbean Studies, 40(2), 124161.

Sikka, P., Filling, S., \& Liew, P. (2009). The audit crunch: reforming auditing. Managerial Auditing Journal, 24(2), 135-155.

Silva, M., Albuquerque, F., Marcelino, M., \& Quirós, J. (2015). A divulgação do risco nas demonstrações financeiras: uma análise ao anexo das sociedades não financeiras portuguesas. Revista de Educação e Pesquisa em Contabilidade, 9(3), 331-354.

Spence, M. (1973). Job market signaling. The Quarterly Journal of Economics, 87(3), 355-374. 
Suárez, J., García, E., Méndez, C., \& Gutiérrez, C. (2013). The effectiveness of audit committee in Spain: implications of its existence on the auditor's opinion. Journal of the Spanish Economic Association. 4(3), 333-351.

Verrecchia, R. (1983). Discretionary disclosure. Journal of Accounting E Economics, 5, 179-194.

Verrecchia, R. E. (1999). Disclosure and the cost of capital: a discussion. Journal of Accounting and Economics, 26, 271-283.

Woods, M., \& Marginson, D. (2004). Accounting for derivatives: an evaluation of reporting practice by UK banks. European Accounting Review, 13(2), 373-390.

Woods, M., Dowd, K., \& Humphrey, C. (2008). Market risk reporting by the world's top banks: evidence on the diversity of reporting practice and the implications for international accounting harmonization. Revista de Contabilidad - Spanish Accounting Review, 11(2), 9-42.

Xavier, P. (2003). Transparência das demonstrações contábeis dos bancos no Brasil: estudo de caso sob a perspectiva do Acordo de 'Basileia 2'. Dissertação de Mestrado em Controladoria e Contabilidade, Universidade de São Paulo.

Yang, R., Yu, Y., Liu, M., \& Wu, K. (2018). Corporate risk disclosure and audit fee: a text mining approach. European Accounting Review, 27(3), 583-594.

Yong, H., Chalmers, K., \& Faff, R. (2005). Asia Pacific banks' derivative and risk management disclosures. Asian Review of Accounting, 13(1), 15-44.

Zango, A., Kamardin, H., \& Ishak, R. (2016). Audit quality, board gender and financial risk disclosure. International Journal of Economics and Financial Issues, 6(4), 55-61.

Zhang, X., Taylor, D., Qu, W., \& Wise, V. (2013). Institutional ownership, audit committee and risk disclosure - evidence from Australian stock market. Corporate Board: Role, Duties \& Composition, $9(3), 66-81$. 